\title{
RESEARCH METHODOLOGY FOR ESTIMATING THE AMPLITUDE-FREQUENCY CHARACTERISTICS OF PIEZOELECTRIC SENSORS BY USING A MECHANICAL SOURCE OF CONTINUOUS NOISE
}

\author{
S. A. Bekher, T. V. Sych* \\ Siberian transport university, 191 D. Kovalchuk st., 630049, Novosibirsk, Russian Federation \\ *Corresponding author. E-mail: tatyna979@mail.ru; address for correspondence: ul. D. Kovalchuk 191, 630049, \\ Novosibirsk, Russian Federation. Tel.:+7 38332803 46; fax: +7 3833280346
}

Experimental results of amplitude-frequency response for acoustic emission sensors are obtained by using a mechanical source of continuous noise. The amplitude-frequency characteristics of a calibrated transducer are analyzed during the registration of longitudinal, surface and Lamb waves. Experimental characteristics of different-type bandpass sensors installed on the object are discussed.

Keywords: acoustic emission test, acoustic emission sensor, calibration, amplitude-frequency characteristic, sensibility.

DOI: $10.17804 / 2410-9908.2016 .2 .014-020$

\section{References}

1. GOST R 52727-2007. Technical Diagnostics. Acoustic-Emission Diagnostics. General Requirements. M., Standartinform Publ., 2007, 18 p. (In Russian).

2. $\quad P B$ 03-593-03. Rules for Organization and Implementation of Acoustic-Emission Testing of Vessels, Apparatuses, Boilers and Process Piping, 2003. (In Russian).

3. Requirements for Acoustic-Emission Transducers Used to Test Hazardous Production Facilities. RD 03-300-99, 01.10.1999, 1999. (In Russian).

4. Stepanova L.N., Grossman S.A., Kabanov S.I., Bobrov A.L., Bekher S.A., Bolchanov A.A. Acoustic-emission testing of solebars with boxlike cross sections. Russian Journal of Nondestructive Testing, vol. 47, no. 3, pp. 158-163. DOI: 10.1134/S1061830911030090.

5. Sych T.V., Gerasimov S.I., Bekher S.A. Acoustic head, RF Patent 152495, 2015. (In Russian).

6. Sych T.V., Bekher S.A., Bobrov A.L. A method for relative calibration of acoustic emission transducers. RF Patent 2554320, 2015, 7 p. (In Russian). 
Подана в журнал: 24.12.2015

УДК 620.179.17

DOI: $10.17804 / 2410-9908.2016 .2 .014-020$

\title{
МЕТОДИКА ИССЛЕДОВАНИЯ ХАРАКТЕРИСТИК ПЬЕЗОЭЛЕКТРИЧЕСКИХ ПРЕОБРАЗОВАТЕЛЕЙ С ИСПОЛЬЗОВАНИЕМ ИСТОЧНИКА ШУМА
}

\author{
С. А. Бехер, Т. В. Сыч*
}

Сибирский государственный университет путей сообщения, 630049, г. Новосибирск, ул. Дуси Ковальчук, 191

*Ответственный автор. Электронная почта: tatyna979@ mail.ru; адрес для переписки: 630049,

г. Новосибирск, ул. Дуси Ковальчук, 191. Телефон: +7 (383) 328-03-46; Факс: +7 (383) 328-03-46

Приведены результаты экспериментальных исследований амплитудно-частотных характеристик преобразователей акустической эмиссии с использованием механического источника непрерывного шума. Проанализированы амплитудно-частотные характеристики калиброванного преобразователя в условиях регистрации продольных, поверхностных и нормальных волн Лэмба. Получены экспериментальные характеристики полосовых преобразователей разных типов, установленных на объекте контроля.

Ключевые слова: акустико-эмиссионный контроль, преобразователь акустической эмиссии, калибровка, амплитудно-частотная характеристика, чувствительность.

\section{1. Введение}

Свойства преобразователей акустической эмиссии определяют основные параметры акустико-эмиссионного контроля: чувствительность, рабочий диапазон частот, помехоустойчивость. В соответствии с требованиями [1] применяемые акустикоэмиссионные системы должны быть калиброваны или поверены в установленном порядке. Проверка работоспособности аппаратуры выполняется непосредственно перед каждым испытанием и после него с использованием импульсного источника Су-Нильсена.

Руководящие документы в области акустико-эмиссионного контроля опасных производственных объектов [2, 3] предъявляют требования к метрологическому обеспечению преобразователей акустической эмиссии. Преобразователи подлежат калибровке не реже одного раза в год и проверке основных параметров перед каждым контролем с использованием эталонных преобразователей. Методика проверки основных параметров подробно описана в [3], при этом процедура определения амплитудно-частотной характеристики (АЧХ) преобразователей не предусмотрена.

На практике спектральный состав регистрируемых импульсов акустической эмиссии определяется АЧХ системы преобразователь - объект контроля. Место расположения преобразователя на поверхности объекта контроля, применяемые волноводы, способ крепления и контактная среда оказывают влияние на АЧХ регистрируемых сигналов акустической эмиссии. В отдельных случаях это влияние может негативно сказываться на достоверности результатов контроля.

В процессе эксплуатации внешние воздействия способны изменять АЧХ преобразователя. В акустико-эмиссионном контроле, а особенно с использованием полосовых и широкополосных преобразователей, процессы проверки работоспособности аппаратуры и определения основных параметров с помощью импульсного источника Су-Нильсена или электронного имитатора не позволяют с высокой точностью оценивать спектральные характеристики системы преобразователь - объект контроля.

Цель исследования - разработка методики оценки амплитудно-частотной характеристики преобразователя акустической эмиссии в реальных условиях эксплуатации с использованием источника акустического шума. 


\section{2. Материал и методика эксперимента}

В стандартных методиках [2, 3] параметры преобразователя оцениваются по импульсной характеристике. Предлагаемая методика основана на регистрации широкополосного акустического сигнала. АЧХ преобразователя оценивалась по спектру регистрируемых сигналов и сравнивалась с паспортными данными эталонного преобразователя. Для сравнения использовали преобразователь GT-300, зарегистрированный в Государственном реестре средств измерений, имеющий действующее свидетельство о поверке. Коэффициент электроакустического преобразования на среднегеометрической частоте 283 кГц при воздействии продольных волн составлял 54 дБ относительно 1 В/(м/с), предельная допускаемая неравномерность АЧХ - не более \pm 5 дБ в диапазоне рабочих частот от 100 до 800 кГц.

Экспериментальные исследования проводили на листе из стали Ст3пс толщиной 8 мм (рис. 1 a). Места установки преобразователей акустической эмиссии зачищали шлифовальной машинкой до шероховатости $\mathrm{Rz}<40$ мкм. Для крепления преобразователей использовали специализированные магнитные держатели [5]. Электрические сигналы регистрировали системой цифровой акустико-эмиссионной диагностической СЦАД-16-03 [4], зарегистрированной в Реестре средств измерений за №39729, с частотой дискретизации 2 МГц. Анализ зарегистрированных сигналов проводили в штатном программном обеспечении диагностической системы и универсальном программном обеспечении MathCad.

В процессе экспериментов исследовали характеристики преобразователей акустической эмиссии типа: ПП 0,1-0,7; ПП-001; GT-300. Акустические сигналы возбуждались трением шлифовального инструмента по поверхности объекта контроля. Насадка из шлифовального материала приводилась во вращение электродрелью с частотой от 10 до 50 Гц. В предварительных экспериментах форма, размер насадки и зернистость поверхности по ГОСТ 3647-80 варьировали в экспериментах с целью получения широкополосного акустического сигнала с равномерным во времени амплитудным распределением. В дальнейшем использовали цилиндрическую насадку диаметром 10 мм с мелкозернистым напылением F80 по ГОСТ Р 52381-2005.

Перед проведением испытаний проводили проверку чувствительности акустикоэмиссионных каналов с помощью имитатора Су-Нильсена по ГОСТ 52727-2007 (рис. 1 a). Наибольшая амплитуда акустико-эмиссионных сигналов (рис. 1 б) зарегистрирована для преобразователя ПП-001 323 мкВ, наименьшая - 69 мкВ для ПП 0,1-0,7. Все преобразователи были подключены к акустико-эмиссионной системе СЦАД-16-03 и находились на равном расстоянии от источника. Сигналы были зарегистрированы практически одновременно, с задержкой по времени прихода не более 2 мкс. Несмотря на одинаковые условия форма сигналов на преобразователях существенно отличалась. 


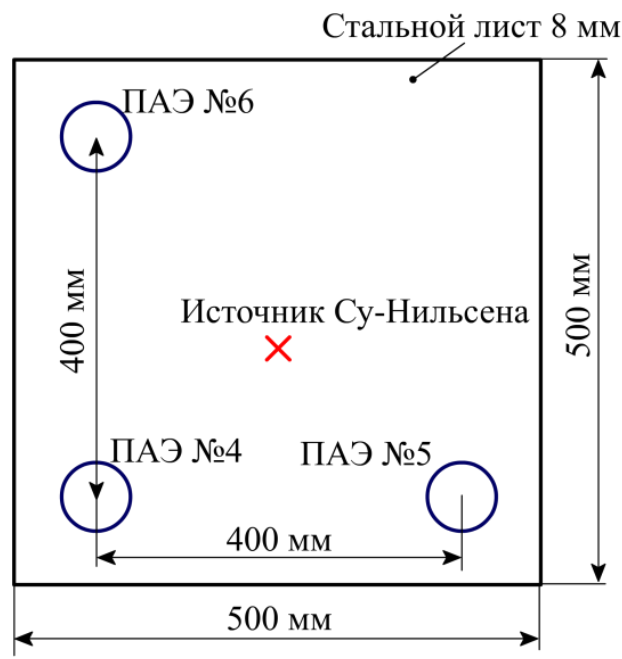

$a$

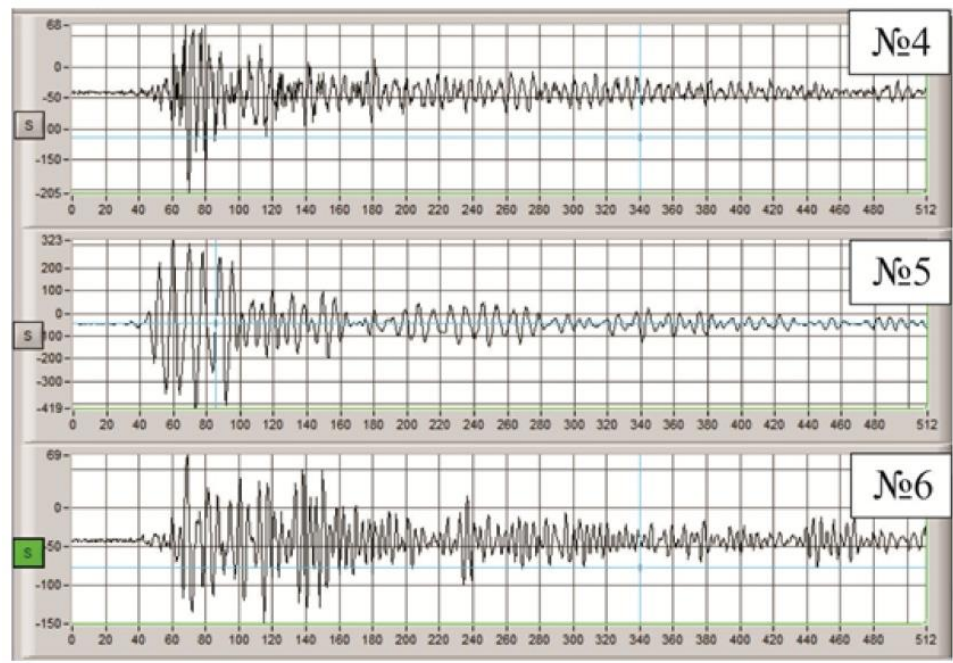

$\sigma$

Рис. 1. Схема установки преобразователей на листе $(a)$ и форма зарегистрированных сигналов (б): ПАЭ № 4 - GT300; ПАЭ № 5 - ПП-001; ПАЭ № 6 - ПП 0,1-0,7

\section{3. Результаты и обсуждение}

В потоке зарегистрированных непрерывных сигналов акустической эмиссии трения отбирали сигналы с постоянной амплитудой, изменение которой на временном интервале 512 мкс не превышало $5 \%$ (рис. 2). АЧХ сигналов (кривая 1, рис. 3) рассчитывалась с использованием алгоритмов быстрого преобразования Фурье и усреднялась по абсолютному значению по 20 сигналам.

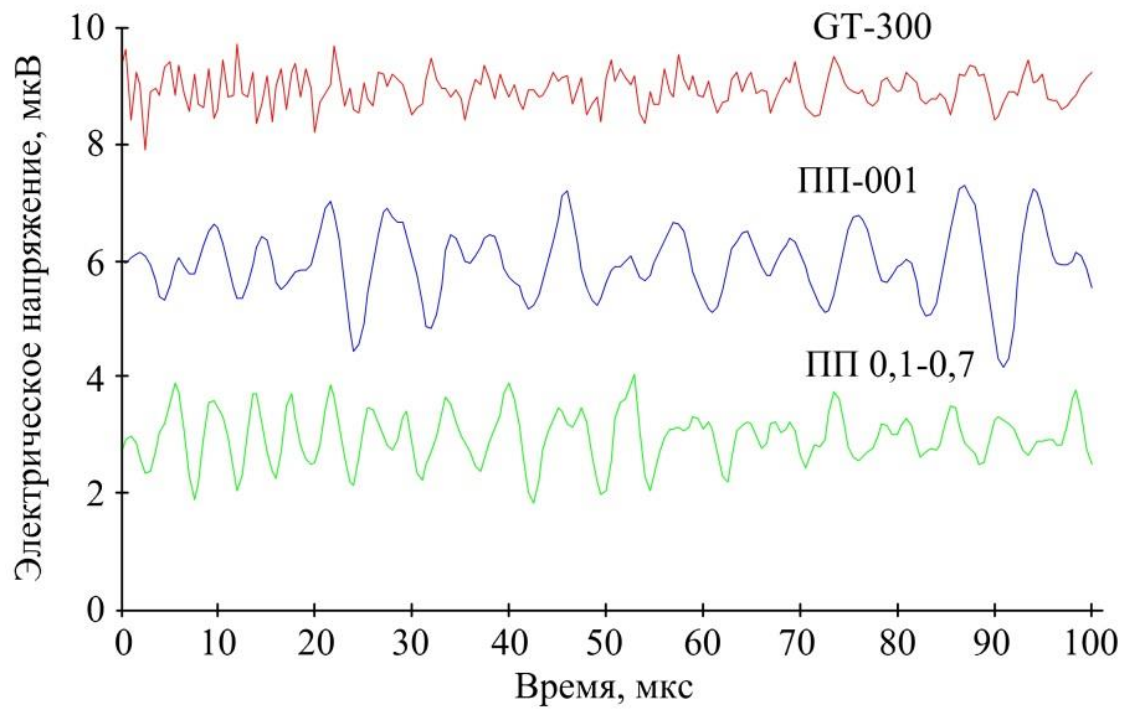

Рис. 2. Сигналы непрерывной акустической эмиссии трения

Для сравнения на рис. 3 приведены две АЧХ (кривые 2 и 3) из паспорта эталонного преобразователя GT-300. Равномерная по уровню 5 дБ АЧХ (кривая 3) получена в условиях регистрации продольных волн, колебания частиц которых перпендикулярны поверхности преобразователя. Неравномерная АЧХ с выраженными экстремумами (кривая 2) на частотах 136, 320, 480, 630 кГц построена по результатам регистрации поверхностных волн. Локальные минимумы на графике АЧХ (кривая 2) связаны с целым числом длин волн, которые укладываются на размере преобразователя. Суммарные деформации пьезопластины и, следовательно, выходной сигнал преобразователя при этом близки к нулю. 
При контроле реальных объектов, изготовленных из листовой стали, акустические сигналы распространяются в виде волн Лэмба, для которых характерно наличие нескольких мод, каждая из которых описывается собственной дисперсионной кривой. Сигналы акустической эмиссии трения вызваны значительным количеством некоррелированных источников акустической эмиссии, что уменьшает эффект от суммирования деформаций на размере пластины. АЧХ зарегистрированных сигналов от механического источника приведен на рис. 3 кривая 1. Локальные минимумы менее выражены и не превышают 10 дБ. В области частот от 600 до 700 кГц наблюдается локальный максимум амплитуд, который также может быть выделен на АЧХ для поверхностных волн из паспорта. В диапазоне частот от 190 до 700 кГц средняя амплитуда АЧХ убывает для поверхностныХ волн с наклоном 0,073 дБ/кГц, для нормальных волн в пластинах с наклоном 0,062 дБ/кГц. Уменьшение связано с эффектом суммирования деформаций пьезопластиной, так как при увеличении частоты уменьшается длина волны и, следовательно, только часть площади пьезопластины участвует в преобразовании колебаний в электрический сигнал.

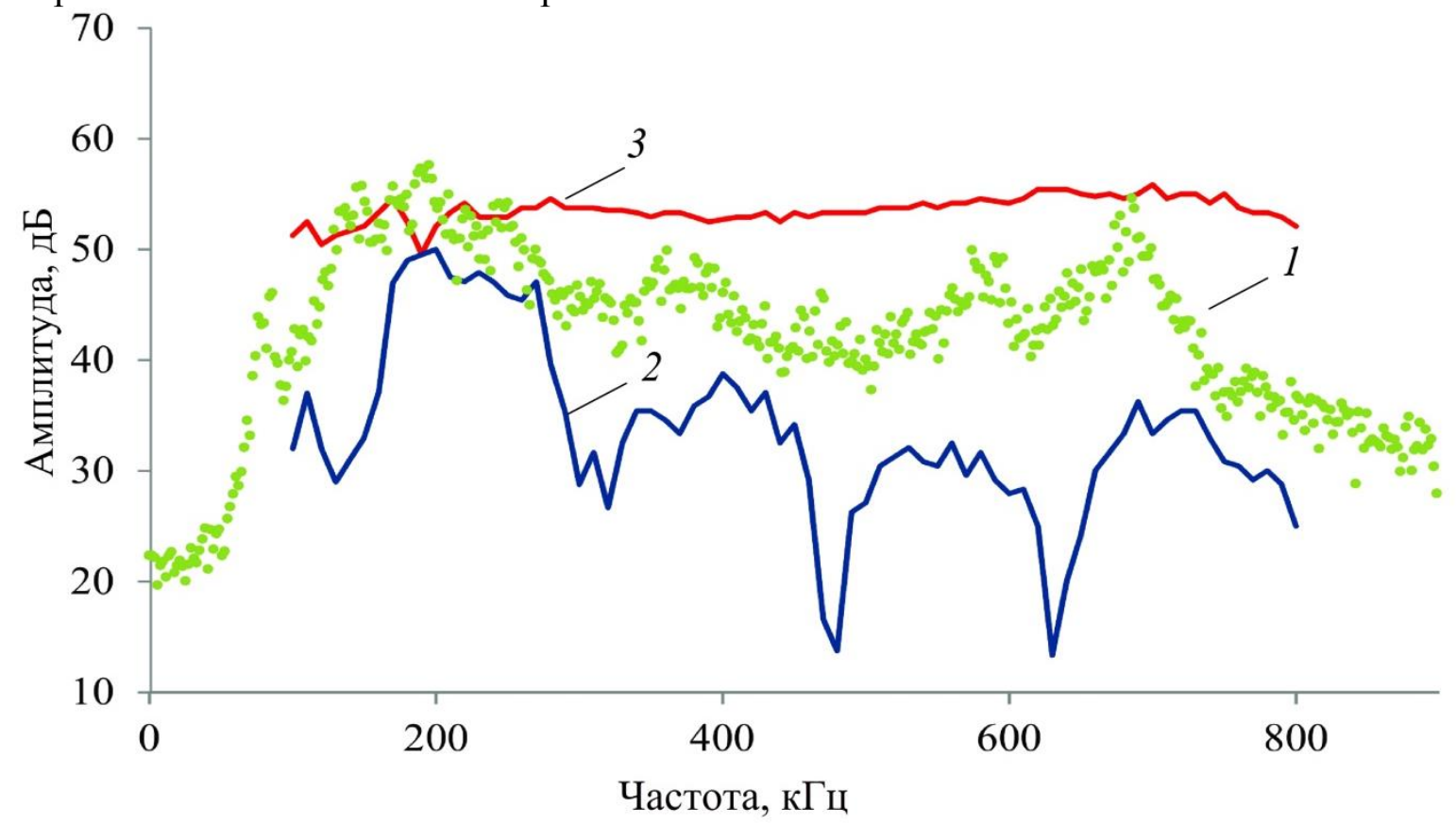

Рис. 3. Амплитудно-частотная характеристика преобразователя GT-300:

1 - экспериментальная; 2 - паспортная для поверхностных волн; 3 - паспортная для продольных волн

На основе предложенного метода оценки АЧХ преобразователей выполнены экспериментальные сравнительные испытания полосовых преобразователей ПП-001 и ПП 0,1-0,7. Для преобразователя ПП-001 амплитудно-частотная характеристика имеет максимум на частотах 120 кГц и 180 кГц, перепад АЧХ для этого преобразователя составляет \pm 15 дБ.

Для преобразователя ПП 0,1-0,7 наблюдаются два пика АЧХ (рис. 4 б) на частотах соответственно 150 кГц и 260 кГц. При этом преобразователь ПП 0,1-0,7 заявлен производителем как полосовой, полученные экспериментальные результаты по предлагаемому способу оценки АЧХ подтверждают эти данные. Перепад амплитудной характеристики для него не превышает \pm 10 дБ. 

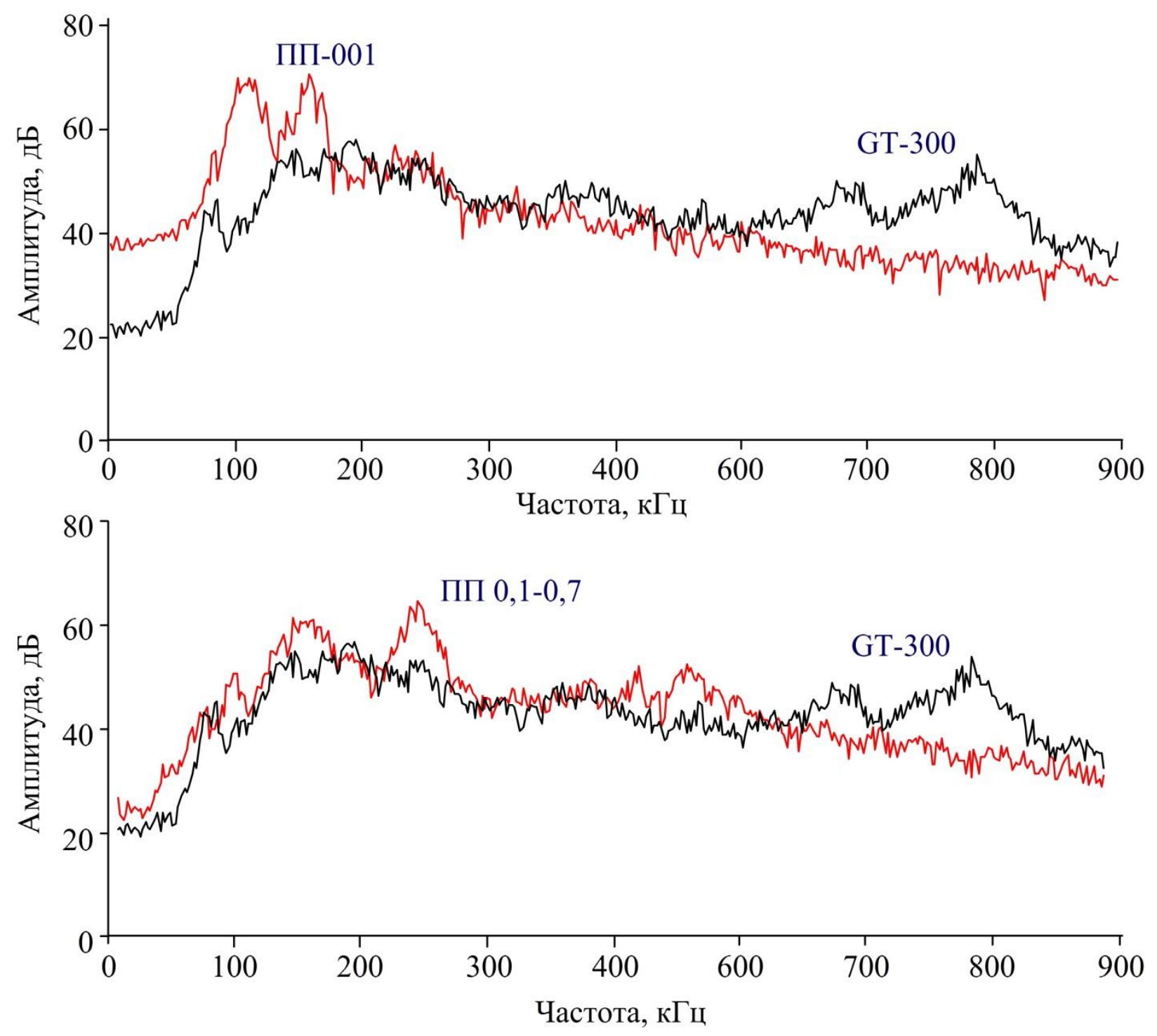

Рис. 4. Амплитудно-частотные характеристики преобразователей

\section{4. Заключение}

Разработанный способ исключает влияние на результаты калибровки преобразователя нестабильности характеристик источника акустического сигнала, исключает влияние на результаты относительной калибровки амплитудно-частотных характеристик образцового преобразователя. Предлагаемая методика проведения калибровки позволяет проводить экспериментальное определение амплитудно-частотной характеристики калибруемого преобразователя за счет получения необходимых экспериментальных данных амплитудного распределения сигналов в широком диапазоне частот. Кроме того, существенным преимуществом является отсутствие жестких требований к геометрии и характеристикам калибровочного блока, что позволит делать калибровку преобразователей в рабочих условиях «на местах», в том числе проводить калибровку преобразователей акустической эмиссии на литых деталях тележки грузового вагона. 


\section{Литература}

1. ГОСТ Р 52727-2007. Техническая диагностика. Акустико-эмиссионная диагностика. Общие требования. Введ. 2007-10-01. - М. : Стандартинформ, 2007. - 18 с.

2. ПБ 03-593-03. Правила организации и проведения акустико-эмиссионного контроля сосудов, аппаратов, котлов и технологических трубопроводов. Утв. 09.06.2003.

3. Требования к преобразователям акустической эмиссии, применяемым для контроля опасных производственных объектов. РД 03-300-99. 01.10.1999. - 1999.

4. Acoustic-emission testing of solebars with boxlike cross sections / L. N. Stepanova, S. A. Grossman, S. I. Kabanov, A. L. Bobrov, S. A. Bekher, A. A. Bolchanov // Russian Journal of Nondestructive Testing. - Vol. 47, no. 3. - P. 158-163. - DOI: 10.1134/S1061830911030090.

5. Акустическая головка : пат. 152495 Рос. Федерация / Сыч Т. В., Герасимов С. И., Бехер С. А., ФГБОУ ВПО Сибирск. гос. универ. путей сообщения. - № 2014150113/28 ; заявл. 10.12.2014 ; опубл. 10.06.2015, Бюл. № 16. - 1 с.: ил.

6. Способ относительной калибровки преобразователей акустической эмиссии: пат. 2554320 Рос. Федерация / Сыч Т. В., Бехер С. А., Бобров А. Л., ФГБОУ ВПО Сибирск. гос. универ. путей сообщения. - № 2014101589/28 ; заявл. 20.01.2014 ; опубл. 27.06.2015, Бюл. № 18. - 7 с.: ил. 